\title{
[gw22-e0406] MICRORNA LET-7I REGULATES LPS-INDUCED MATURATION AND IMMUNE INDUCTION BY DENDRITIC CELLS THROUGH TRANSLATIONAL REPRESSION OF SOCS1
}

Zhang Maomao, Wu Jian, Liu Fang, Zhang Qi, Yu BoSecond Affiliated Hospital Of Harbin Medical University, Harbin, China

\subsection{6/heartjnl-2011-300867.44}

Dendritic cells (DCs) can initiate immune responses or confer immune tolerance depending on functional status. Lipopolysaccharide (LPS) induced DC maturation as defined by enhanced surface expression of CD80 and CD86. MicroRNAs (miRNAs) are critical for the regulation of DC function and immunity, and the miRNA let-7i was up-regulated during LPS-induced DC maturation. Down-regulation of let-7i significantly impeded DC maturation as evidenced by reduced CD80 and CD86 expression. DCs stimulated by LPS (LPS-DCs) promoted T-cell proliferation in co-culture, while LPS-stimulated DCs with down-regulated let-7i were not effective at stimulating $T$ cell proliferation but promoted expansion of the regulatory $T$ cell (Treg) population. There were two subpopulations of LPS-stimulated DCs with downregulated let-7i, $\mathrm{CD} 86^{-}$and $\mathrm{CD} 86^{+}$, and it was the $\mathrm{CD} 86^{-} \mathrm{DCs}$ that were more effective in inducing $\mathrm{T}$ cell hyporesponsiveness and enhancing Treg numbers, indicating that this DC population had tolerogenic properties. Furthermore, Treg with up-regulated IL-10 underscored the tolerogenic effect of CD86- DCs. Suppressor of cytokine signalling 1 (SOCS1), a crucial mediator of DC maturation, was confirmed as a let-7i target gene by luciferase construct assay. Suppression or overexpression of let-7i caused reciprocal alterations in SOCS1 protein expression, but had no significant effects on SOCS1 mRNA levels, indicating that let-7i regulated SOCS1 expression by translational suppression. The modulation of SOCS1 protein by let-7i was mainly restricted to CD86- DCs. Our study demonstrates that let-7i regulation of SOCS1 is critical for LPS-induced DC maturation and immune function. Dynamic regulation of let-7i may fine tune immune responses by inducing antigen-specific immune tolerance. 